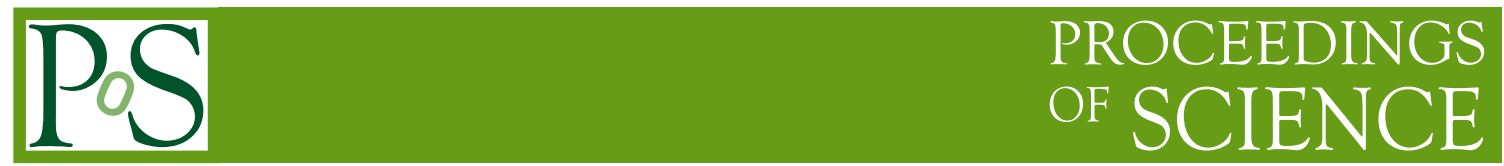

\title{
Heavy flavor production in the forward acceptance at the LHC
}

\section{Ivan Polyakov*i}

Institute for Theoretical and Experimental Physics, Moscow, Russia

E-mail: ivan.polyakovecern.ch

In this report the most recent results on heavy flavor production studies at the LHCb experiment are covered. The measurements of production cross-sections of the $\chi_{b J}(\mathrm{mP}), \eta_{c}(1 \mathrm{~S})$ and $B_{c}^{+}$ states in proton-proton collisions at center of mass energies $\sqrt{s}=7$ and $8 \mathrm{TeV}$ are presented.

The European Physical Society Conference on High Energy Physics

22-29 July 2015

Vienna, Austria

*Speaker.

On behalf of the LHCb collaboration. 


\section{Introduction}

Heavy flavor and quarkonia production is a long standing puzzle of QCD. Its various aspects have been successfully described by several theoretical approaches (color-octet and color-singlet mechanisms, etc. [1, 2, 3, 4]). However, the relative role of these proposed mechanisms is poorly understood. The discrepancies between data and theory predictions tend to become more visible in the region of small transverse momenta and large rapidities. At the LHC, the LHCb experiment [5] stands in this region having a coverage of $2<\eta<5$ and therefore providing an unique possibility for stringent test of the theory. The previous studies of the heavy flavor production at the LHCb experiment have covered the following topics: charmonium $\left(J / \psi, \psi(2 S), \chi_{c 0,1,2}\right.$ and $\left.X(3872)\right)$ and bottomonium $\left(\Upsilon(\mathrm{nS}), \chi_{b}(1 \mathrm{P})\right)$ production in $p p$ - and $p P b$-collisions, $b$-hadron $\left(B^{+/ 0}, B_{s}^{0}, B_{c}^{+}\right.$and $\left.\Lambda_{b}^{0}\right)$ production and also double charm production $\left(J / \psi J / \psi, J / \psi D\right.$ and $D D$, where $D$ is $D^{+/ 0}, D_{s}^{+}$ or $\Lambda_{c}^{+}$). In this report, the recent results of these studies are presented.

\section{Production of $\chi_{b J}(\mathrm{mP})$ states}

Investigation of $\chi_{b J}(\mathrm{mP})$ states is performed using $\chi_{b J}(\mathrm{mP}) \rightarrow \Upsilon(\mathrm{nS}) \gamma$ decays where the photon is reconstructed either in the electromagnetic calorimeter [6] or through its conversion into $e^{+} e^{-}$pair in the detector [7]. The $\Upsilon(\mathrm{nS})$ mesons are reconstructed via their dimuon decays and selected by requiring two tracks identified as muons, having large transverse momentum and common vertex compatible with the primary $p p$-interaction vertex. The events with dimuon invariant mass compatible with $\Upsilon(1,2,3 \mathrm{~S})$ states are then combined with photon candidates having transverse momentum larger than $0.6 \mathrm{GeV} / \mathrm{c}$ to form $\chi_{b J}(\mathrm{mP})$ candidates. When reconstructing a photon through its conversion to $e^{+} e^{-}$, invariant mass of the dielectron pair is required to be less than $50 \mathrm{MeV} / \mathrm{c}^{2}$.

Invariant mass distributions of selected $\Upsilon(\mathrm{nS}) \gamma$ candidates with photon reconstructed in electromagnetic calorimeter are presented in Fig. 1 showing various $\chi_{b J}(\mathrm{mP}) \rightarrow \Upsilon(\mathrm{nS}) \gamma$ transitions. Based on these signals the fractions of $\Upsilon(\mathrm{nS})$ from feed-down

$$
R_{\Upsilon(\mathrm{nS})}^{\chi_{b}(\mathrm{mP})} \equiv \frac{\sigma\left(p p \rightarrow \chi_{b 1}(\mathrm{mP}) X\right)}{\sigma(p p \rightarrow \Upsilon(\mathrm{nS}) X)} \times \mathscr{B}_{1}+\frac{\sigma\left(p p \rightarrow \chi_{b 2}(\mathrm{mP}) X\right)}{\sigma(p p \rightarrow \Upsilon(\mathrm{nS}) X)} \times \mathscr{B}_{2},
$$

are measured, where $\mathscr{B}_{1,2}$ are the $\chi_{b 1,2}(\mathrm{mP}) \rightarrow \Upsilon(\mathrm{nS}) \gamma$ branching fractions. The feed-down is found to be of order of $30 \%$ and show no dependence on $p p$-collision energy. The fraction $R_{\Upsilon(3 \mathrm{~S})}^{\chi_{b}(3 \mathrm{P})}$ is measured for the first time and found to be unexpectedly large. In addition, using the
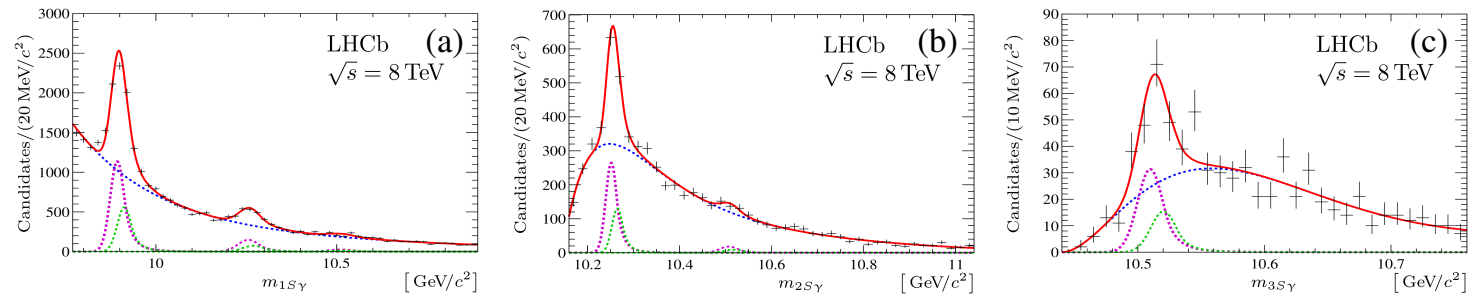

Figure 1: Invariant mass distributions of (a) $\Upsilon(1 S) \gamma$, (b) $\Upsilon(2 S) \gamma$ and (c) $\Upsilon(3 S) \gamma$ candidates with photon reconstructed in electromagnetic calorimeter. The data collected at energy of $\sqrt{s}=8 \mathrm{TeV}$ are shown. 

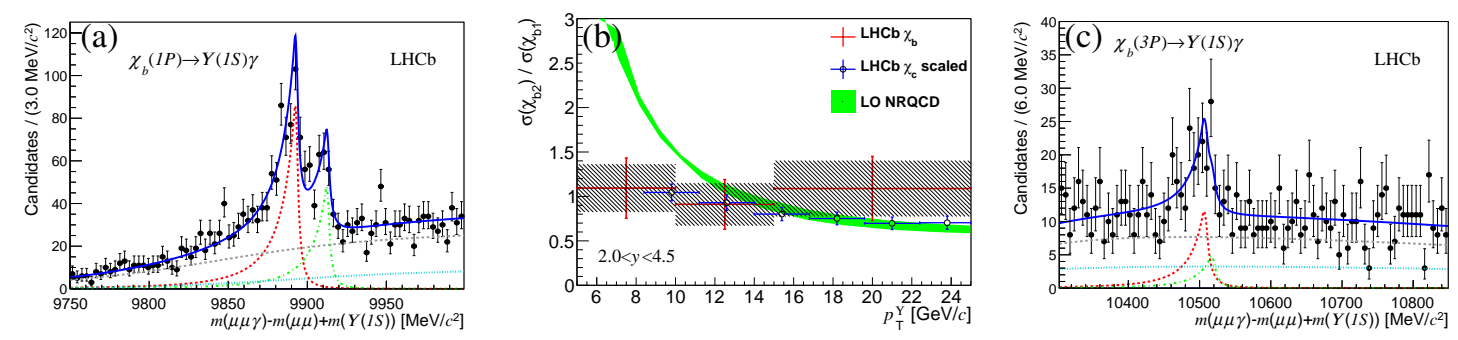

Figure 2: (a) Invariant mass distribution of $\chi_{b}(1 \mathrm{P}) \rightarrow \Upsilon(1 \mathrm{~S}) \gamma$ candidates with photon reconstructed through conversion to dielectron pair. (b) Relative production cross-sections of $\chi_{b 1}(1 \mathrm{P})$ and $\chi_{b 2}(1 \mathrm{P})$ mesons as function of $p_{\mathrm{T}}^{\Upsilon}$. (c) Invariant mass distribution of $\chi_{b}(3 \mathrm{P}) \rightarrow \Upsilon(1 \mathrm{~S}) \gamma$ candidates with photon reconstructed through conversion to dielectron pair.

$\chi_{b J}(3 \mathrm{P}) \rightarrow \Upsilon(3 \mathrm{~S}) \gamma$ decay the mass of the $\chi_{b 1}(3 \mathrm{P})$ state is measured to be $m_{\chi_{b 1}(3 \mathrm{P})}=10511.3 \pm$ 1.7 (stat) \pm 2.5 (syst) $\mathrm{MeV} / \mathrm{c}^{2}$. This result is compatible and more precise than the measurements reported previously by the ATLAS [8] and D0 [9] experiments.

Owing to the good energy resolution obtained with converted photons, the $\chi_{b 1}(\mathrm{mP})$ and $\chi_{b 2}(\mathrm{mP})$ states could be resolved as shown in Fig. 2(a). Thus, the production cross section of $\chi_{b 2}(1 \mathrm{P})$ relative to $\chi_{b 1}(1 \mathrm{P})$ is measured for the first time. Its $p_{\mathrm{T}}$ dependency is shown in the Fig. 2(b). The results agree with LO NRQCD predictions [10], theory expectation based on LHCb $\chi_{c}$ measurements [11] and also with CMS results [12]. The $\chi_{b J}(3 \mathrm{P}) \rightarrow \Upsilon(1 \mathrm{~S}) \gamma$ and $\chi_{b J}(3 \mathrm{P}) \rightarrow \Upsilon(2 \mathrm{~S}) \gamma$ decays are also observed (see Fig. 2(c)) and used to measure the mass of the $\chi_{b 1}(3 \mathrm{P})$ state to be $m_{\chi_{b 1}(3 \mathrm{P})}=10515.7_{-3.9}^{+2.2}(\text { stat })_{-2.1}^{+1.5}($ syst $) \mathrm{MeV} / \mathrm{c}^{2}$.

\section{Production of $\eta_{c}(1 S)$ state}

Investigation of $\eta_{c}(1 \mathrm{~S})$ states complements the previous studies of $J / \psi, \psi(2 \mathrm{~S})$ and $\chi_{c 0,1,2}(1 \mathrm{P})$ production performed at $\mathrm{LHCb}$. Measurement of its production cross-section, in particular in the low $p_{\mathrm{T}}$ region, can have important implications. It allows to test heavy-quark spin-symmetry relation between the $\eta_{c}(1 \mathrm{~S})$ and $J / \psi$ matrix elements as the NLO NRQCD calculations predict different $p_{\mathrm{T}}$ dependence of the production rates for spin singlet $\left(\eta_{c}(1 \mathrm{~S}), h_{c}(1 \mathrm{P})\right)$ and spin triplet $\left(J / \psi, \chi_{c 0,1,2}(1 \mathrm{P})\right)$ states $[13,14,15]$.

In the analysis performed by the LHCb experiment [16] the $\eta_{c}(1 \mathrm{~S})$ and $J / \psi$ states are reconstructed via their decays to $p \bar{p}$ pair, which could be done thanks to excellent proton identification provided by the LHCb detector. The pseudo-proper decay time defined as $t_{z}=\Delta z M / p_{z}$ (where $M$ and $p_{z}$ are invariant mass and longitudinal momentum of the $p \bar{p}$ pair respectively) is used to separate prompt $\eta_{c}(1 \mathrm{~S})$ production from production in $b$-decays. Invariant mass distribution of $p \bar{p}$ candidates produced in $b$-decays is shown in Fig. 3(a). In the figure, the peaks corresponding to $\eta_{c}(1 \mathrm{~S})$ and $J / \psi$ states are clearly seen. The fit of this distribution gives the following $\eta_{c}(1 \mathrm{~S})$ and $J / \psi$ events yields: $N_{\eta_{c}(1 \mathrm{~S})}^{b}=2020 \pm 230$ and $N_{J / \psi}^{b}=6110 \pm 116$. The efficiencies for $\eta_{c}(1 \mathrm{~S})$ and $J / \psi$ modes are the same within $0.5 \%$. Therefore, neglecting the bias due to requirement on $p_{\mathrm{T}}(p \bar{p})>6.5 \mathrm{GeV} / \mathrm{c}$, the ratio of yields can be easily transformed into the following ratio of branching fractions: 

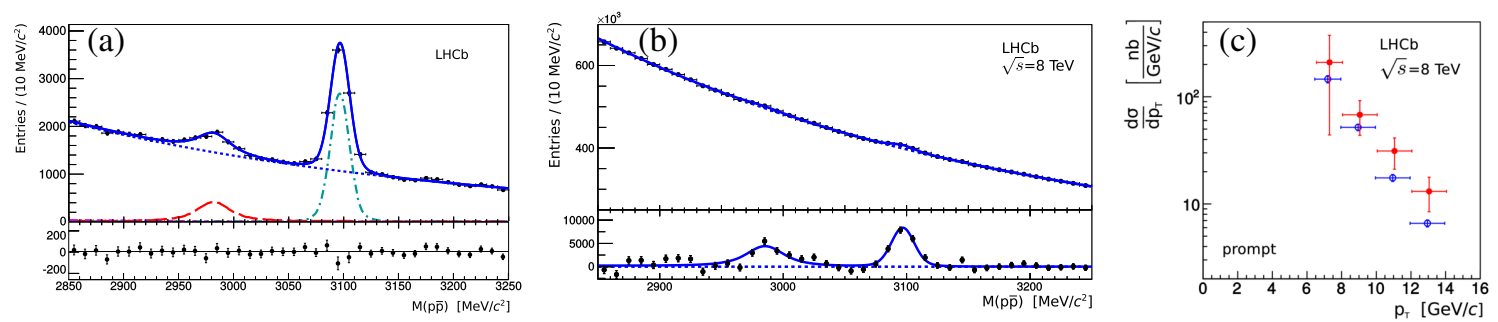

Figure 3: Invariant mass distribution of $p \bar{p}$ candidates produced in (a) $b$-decays and (b) in primary $p p$ collisions. (c) Prompt production spectra of $\eta_{c}(1 \mathrm{~S})$ mesons (red filled circles) in comparison with spectra of $J / \psi$ mesons.

$$
\frac{\mathscr{B}\left(b \rightarrow \eta_{c}(1 \mathrm{~S}) X\right)}{\mathscr{B}(b \rightarrow J / \psi X)}=\frac{N_{\eta_{c}(1 \mathrm{~S})}^{b}}{N_{J / \psi}^{b}} \times \frac{\mathscr{B}(J / \psi \rightarrow p \bar{p})}{\mathscr{B}\left(\eta_{c}(1 \mathrm{~S}) \rightarrow p \bar{p}\right)}=0.421 \pm 0.055(\text { stat }) \pm 0.025(\text { syst }) \pm 0.045(\mathscr{B})
$$

This is the first measurement of the branching fraction of inclusive $b$-decays to $\eta_{c}(1 \mathrm{~S})$ and it agrees with upper limit of $\mathscr{B}\left(B^{-}, \bar{B}^{0} \rightarrow \eta_{c}(1 \mathrm{~S}) X\right)<9 \times 10^{-3}$ at $90 \%$ previously reported by the CLEO collaboration [17].

The parameters of $\eta_{c}(1 S)$ and $J / \psi$ peaks, determined from the fit with candidates from $b$ decays, are used in the fit of invariant mass distributions of $p \bar{p}$ candidates produced in primary $p p$-collisions (see Fig. 3(b)). The fits yields are $13370 \pm 2260 \eta_{c}(1 \mathrm{~S})$ and $11052 \pm 1004 \mathrm{~J} / \psi$ signal decays for the data taken at $\sqrt{s}=7 \mathrm{TeV}$, and 22416 $\pm 4072 \eta_{c}(1 \mathrm{~S})$ and $20217 \pm 1403 \mathrm{~J} / \psi$ signal decays for the $\sqrt{s}=8 \mathrm{TeV}$ data. Based on these yields, the prompt production cross-sections of $\eta_{c}(1 \mathrm{~S})$ state in kinematic region of $2<y<4.5$ and $p_{\mathrm{T}}>6.5 \mathrm{GeV} / \mathrm{c}$ at $\sqrt{s}=7$ and $8 \mathrm{TeV}$ are determined relatively to $J / \psi$ :

$$
\begin{aligned}
& \left(\sigma_{\eta_{c}(1 \mathrm{~S})} / \sigma_{J / \psi}\right)_{\sqrt{s}=7 \mathrm{TeV}}=1.74 \pm 0.29(\text { stat }) \pm 0.28(\text { syst }) \pm 0.18(\mathscr{B}), \\
& \left(\sigma_{\eta_{c}(1 \mathrm{~S})} / \sigma_{J / \psi}\right)_{\sqrt{s}=8 \mathrm{TeV}}=1.60 \pm 0.29(\text { stat }) \pm 0.25(\text { syst }) \pm 0.17(\mathscr{B}) .
\end{aligned}
$$

Using previously measured production cross-sections of $J / \psi$ as a reference, the $\eta_{c}(1 \mathrm{~S})$ production cross-sections are determined to be

$$
\begin{aligned}
& \left(\sigma_{\eta_{c}(1 \mathrm{~S})}\right)_{\sqrt{s}=7 \mathrm{TeV}}=0.52 \pm 0.09(\text { stat }) \pm 0.08(\text { syst }) \pm 0.06\left(\sigma_{J / \psi}, \mathscr{B}\right), \\
& \left.\left(\sigma_{\eta_{c}(1 \mathrm{~S})}\right)_{\sqrt{s}=8 \mathrm{TeV}}=0.59 \pm 0.11 \text { (stat }\right) \pm 0.09(\text { syst }) \pm 0.08\left(\sigma_{J / \psi}, \mathscr{B}\right) .
\end{aligned}
$$

This is the first measurement of $\eta_{c}(1 \mathrm{~S})$ prompt production in $p p$-collisions. The results are consistent with predictions of color singlet leading order calculations [18]. The $\eta_{c}(1 \mathrm{~S})$ differential cross-section as a function of $p_{\mathrm{T}}$ is also measured (see Fig. 3(c)). In contrast to NLO NRQCD expectations no difference in $p_{\mathrm{T}}$ dependence between $\eta_{c}(1 \mathrm{~S})$ and $J / \psi$ is observed.

\section{Production of $B_{c}^{+}$meson}

The $B_{c}^{+}$meson is a unique quarkonium system being composed of two heavy quarks or different flavor $(\bar{b}$ and $c)$. Besides of having similarities with both charmonium and bottomonium systems it has its own specific features. At the LHC, the $B_{c}^{+}$mesons are mainly produced through 

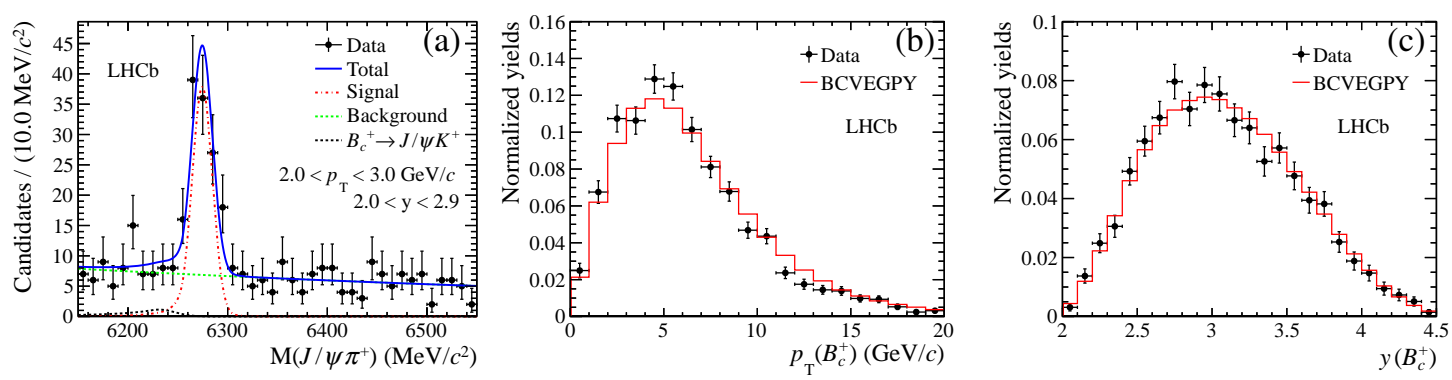

Figure 4: (a) Invariant mass distribution of $B_{c}^{+}$candidates in one of the kinematic regions. Backgroundsubtracted (b) $p_{\mathrm{T}}$ and (c) $y$ distributions of $B_{c}^{+}$mesons. The red line represents the spectra obtained with the simulation.

gluon-gluon fusion $g g \rightarrow(b \bar{b})(c \bar{c}) \rightarrow B_{c}^{+}+b+c$ with leading contribution of order of $\alpha_{s}^{4}$ [19] giving three order suppression with respect to $B^{+/ 0}$ mesons. The main contribution to the $B_{c}^{+}$ production comes from two competitive mechanisms: fragmentation and recombination, having different impact on $p_{\mathrm{T}}$ spectra (also different from spectra of $B^{+/ 0}$ mesons). Therefore it is particularly important to extend the previous measurements by LHCb and CMS experiments of the ratio $R_{c / u}$ defined as

$$
R_{c / u}=\frac{\sigma\left(B_{c}^{+}\right) \mathscr{B}\left(B_{c}^{+} \rightarrow J / \psi \pi^{+}\right)}{\sigma\left(B^{+}\right) \mathscr{B}\left(B^{+} \rightarrow J / \psi K^{+}\right)}
$$

to a measurement of its kinematic dependence.

In the recent analysis performed by the LHCb experiment [20] with data collected at energy $\sqrt{s}=8 \mathrm{TeV}$ the decays $B_{c}^{+} \rightarrow J / \psi \pi^{+}$and $B^{+} \rightarrow J / \psi K^{+}$with similar kinematics and topology are used. The $B_{c}^{+}$and $B^{+}$mesons are reconstructed in $2<y<4.5$ and $0<p_{\mathrm{T}}<20 \mathrm{GeV} / \mathrm{c}$ kinematic region and selected with help of the boost decision trees (BDT) method. The invariant mass of the selected $B_{c}^{+}$candidates in a reduced kinematic region is shown in Fig. 4(a). Based on the fits to invariant mass distributions in various kinematic regions approximately $3100 \mathrm{~B}_{c}^{+}$mesons are observed and their background-subtracted $p_{\mathrm{T}}$ and $y$ spectra are determined (see Fig. 4(b,c)). The figure shows the remarkable fact that the simulation, based on BCVEGPY generator [21], provides a good description of $p_{\mathrm{T}}$ and $y$ distributions of $B_{c}^{+}$mesons in data. The ratio $R_{c / u}$ integrated over the whole $2<y<4.5$ and $0<p_{\mathrm{T}}<20 \mathrm{GeV} / \mathrm{c}$ kinematic region is measured to be

$$
R_{c / u}=(0.683 \pm 0.018 \text { (stat) } \pm 0.009 \text { (syst) }) \%,
$$

which is consistent with the previous measurement at $\sqrt{s}=7 \mathrm{TeV}$. For the first time the doubledifferential production cross-section of $B_{c}^{+}$meson is measured with respect to $B^{+}$. The dependence of this ratio $R_{c / u}$ on kinematics is observed as shown in Fig. 5. These results will provide useful information on the $B_{c}^{+}$production mechanism, help to understand the quarkonium production and therefore deepen our understanding of QCD.

\section{Summary}

The LHCb experiment provides unique input for investigation of QCD by exploiting heavy flavor production in the forward region at the LHC. The production cross-sections of $\chi_{b}(\mathrm{mP})$, 

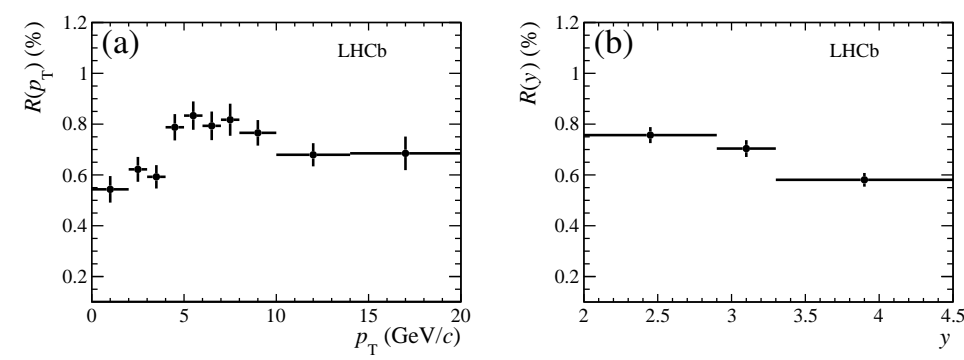

Figure 5: Ratio $R_{c / u}$ as a function (a) of $p_{\mathrm{T}}$ integrated over $y$ in the region $2<y<4.5$ and (b) of $y$ integrated over $p_{\mathrm{T}}$ in the region $0<p_{\mathrm{T}}<20 \mathrm{GeV} / \mathrm{c}$.

$\eta_{c}(1 \mathrm{~S})$ and $B_{c}^{+}$states are recently measured providing new tests for the theory. This measurements show a great potential of the $\mathrm{LHCb}$ experiment to obtain even more exciting results with future data at energy $\sqrt{s}=13 \mathrm{TeV}$.

\section{References}

[1] E.L. Berger and D. Jones, Inelastic photoproduction of J/ $\psi$ and $\Upsilon$ by gluons, PRD 23 (1981) 1521.

[2] R. Baier and R.Ruckl, Hadronic production of $J / \psi$ and $\Upsilon$ : transverse momentum distributions, PLB 102 (1981) 364.

[3] E. Braaten and S. Fleming, Color octet fragmentation and the $\psi$ surplus at the Fermilab Tevatron, PRL 74 (1995) 3327.

[4] G.T. Bodwin, E. Braaten, and G.P. Lepage, Rigorous QCD analysis of inclusive annihilation and production of heavy quarkonium, PRD 51 (1995) 1125.

[5] A.A. Alves Jr. et. al (LHCb Collaboration), The LHCb detector at the LHC, JINST 3 (2008) S08005.

[6] R. Aaij et. al (LHCb Collaboration), Study of $\chi_{b}$ meson production in pp collisions at $\sqrt{s}=7$ and $8 \mathrm{TeV}$ and observation of the decay $\chi_{b} \rightarrow \Upsilon(3 \mathrm{~S}) \gamma$, EPJC 74 (2014) 3092.

[7] R. Aaij et. al (LHCb Collaboration), Measurement of the $\chi_{b}(3 \mathrm{P})$ mass and of the relative rate of $\chi_{b 1}(1 \mathrm{P})$ and $\chi_{b 2}(1 \mathrm{P})$ production, JHEP 10 (2014) 088.

[8] G. Aad et. al (ATLAS Collaboration), Observation of a new $\chi_{b}$ state in radiative transitions to $\Upsilon(1 \mathrm{~S})$ and $\Upsilon(2 S)$ at ATLAS, PRL 108 (2012) 152001.

[9] V.M. Abazov et. al (D0 Collaboration), Observation of a narrow mass state decaying into $\Upsilon(1 \mathrm{~S})+\gamma$ in $p \bar{p}$ collisions at $\sqrt{s}=1.96 \mathrm{TeV}, \mathrm{PRD} 86$ (2012) 031103.

[10] A.K. Likhoded, A.V. Luchinsky and S.V. Poslavsky, Production of $\chi_{b}$-mesons at LHC, PRD 86 (2012) 074027.

[11] R. Aaij et. al (LHCb Collaboration), Measurement of the relative rate of prompt $\chi_{c 0}, \chi_{c 1}$ and $\chi_{c 2}$ production at $\sqrt{s}=7 \mathrm{TeV}, \mathrm{JHEP} \mathbf{1 0}$ (2013) 115.

[12] V. Khachatryan et. al (CMS Collaboration), Measurement of the production cross section ratio $\sigma\left(\chi_{b 2}(1 \mathrm{P})\right) / \sigma\left(\chi_{b 1}(1 \mathrm{P})\right)$ in pp collisions at $\sqrt{s}=8 \mathrm{TeV}$, PLB 743 (2015) 383.

[13] F. Maltoni and A. Polosa, Observation potential for $\eta_{b}$ at the Fermilab Tevatron, PRD 70 (2004) 054014.

[14] A. Petrelli et. al, NLO production and decay of quarkonium, NPB 514 (1998) 245. 
[15] J.H. Kuhn and E. Mirkes, QCD corrections to toponium production at hadron colliders, PRD 48 (1993) 179.

[16] R. Aaij et. al (LHCb Collaboration), Measurement of the $\eta_{c}(1 \mathrm{~S})$ production cross-section in proton-proton collisions via the decay $\eta_{c}(1 \mathrm{~S}) \rightarrow p \bar{p}$, EPJC 75 (2015) 311.

[17] R. Balest et. al (CLEO collaboration), Inclusive decays of B mesons to charmonium, PRD 52 (1995) 2661.

[18] S. S. Biswal and K. Sridhar, $\eta_{c}$ production at the Large Hadron Collider, JPG 39 (2012) 015008.

[19] A.V. Berezhnoy, A.K. Likhoded, M.V. Shevlyagin, Hadronic production of B-mesons, Phys. Atom. Nucl. 58 (1995) 672, arXiv:hep-ph/9408284.

[20] R. Aaij et. al (LHCb Collaboration), Measurement of $B_{c}^{+}$production in proton-proton collisions at $\sqrt{s}=8 T e V$, PRL 114 (2015) 132001.

[21] C.-H. Chang, J.-X. Wang, and X.-G. Wu, BCVEGPY2.0: An upgraded version of the generator $B C V E G P Y$ with the addition of hadroproduction of the P-wave $B_{c}$ states, CPC 174 (2006) 241. 